\title{
Rapid decolorization of water soluble azo-dyes by nanosized zero-valent iron immobilized on the exchange resin
}

\author{
ZHAO ZongShan ${ }^{1}$, LIU JingFu' ${ }^{1}$, TAI Chao $^{1}$, ZHOU QunFang $^{1}$, HU JingTian $^{2}$ \& JIANG GuiBin ${ }^{1 \dagger}$ \\ ${ }^{1}$ Key Laboratory of Environmental Chemistry and Ecotoxicology, Research Center for Eco-Environmental Sciences, Chinese \\ Academy of Sciences, Beijing 100085, China; \\ ${ }^{2}$ Department of Chemistry, Shandong University, Jinan 250100, China
}

\begin{abstract}
Nanosized zero-valent iron (NZVI) supported on the cation exchange resin was synthesized and applied to decompose some water soluble azo dyes. The decomposition efficiency for azo dyes was evaluated by using the aqueous suspensions and parked column of this material. Batch experiments indicated that this novel material exhibited excellent degradation ability for $0.05 \mathrm{~g} \cdot \mathrm{L}^{-1}$ of Acid Orange 7, Acid Orange 8, Acid Orange 10, Sunset Yellow, and Methyl Orange, with decolorization ratio up to $95 \%$ in 4 $\min ; \mathrm{pH}$ value was the key factor for degradation and $\mathrm{H}^{+}$was one of the reactants; adsorption of azo dyes onto the material existed at the beginning but reduced gradually until disappearing completely. For the packed column system, 58\% $90 \%$ of azo dyes were decomposed in the 1st circle of solution passing through the column, and the adsorption onto the materials could accelerate the degradation azo dyes with the increasing reaction time. During the degradation process, $\mathrm{Fe}^{2+}$, the product of NZVI, was exchanged to the resin again and could be reduced to $\mathrm{Fe}^{0}$ by $\mathrm{KBH}_{4}$ for reusing. The 10th refreshed NZVI possessed reductive activity up to $90 \%$ of the newly systhesized NZVI. Decomposing pollutants in the aqueous solution with columns packed with NZVI immobilized on the cation exchange resin is a promising technology that can solve the reclaiming and refreshing problem of NZVI.
\end{abstract}

azo-dye, nanosized-zero valent iron (NZVI), decomposition, packing column

Azo dyes, widely used in the textile and dyestuff industries, account for two-thirds of the organic colorants listed in the Colour Index if colorant precursors and sulfur dyes of indeterminate constitution are excluded. They are often designed to resist the breakdown of long-term exposure to sunlight, water, soap, and other atrocious conditions. Due to the formation of carcinogenic amines when used with textiles for body-contact end-uses, some azo dyes have been banned by some European countries in the last decades. However, many acid and direct dyes that may liberate amines, such as benzidine, $o$-tolidine, and $o$-dianisidine, are still used. The releasing of these dyes to the environment can cause acute and/or chronic effects on the exposed organisms, adsorb or reflect sunlight entering into water, and thus result in change of food chain. Up to now, the treatment methods for azo dyes can be divided into 3 categories: (1) physical methods ${ }^{[1,2]}$, such as flocculation, adsorption, nanofiltration and ion exchange; (2) chemical methods ${ }^{[3-5]}$, such as oxidative process with Fenton's reagent, $\mathrm{H}_{2} \mathrm{O}_{2}$ ozone, $\mathrm{H}_{2} \mathrm{O}_{2}$-UV radiation, $\mathrm{NaClO}$ and $\mathrm{O}_{3}$, and so on; and (3) biological degradation ${ }^{[6]}$, including aerobic and anaerobic degradation. Physical methods are unable to absolutely decompose azo dyes, and chemical methods with high cost are rarely used in the actual treatment process. Biodegradation processes, the most widely used

\footnotetext{
Received March 21, 2007; accepted May 23,2007

doi: $10.1007 / \mathrm{s} 11426-007-0121-\mathrm{x}$

Corresponding author (email: gbjiang@rcees.ac.cn)

Supported by the National Natural Science Foundation of China (Grant No
} 20537020) 
methods suffered from low degradation efficiency or even no degradation for some dyes, and the requirement of a large volume of treatment equipment, so the combination of pretreatment processes, which transform azo the dyes into easier bio-decomposition compounds, with biodegradation process is preferred ${ }^{[7]}$.

Zero valent iron (ZVI), an environmentally friendly strong reducing agent, has been widely studied for environmental remediation in recent years. Now, it has been used in treating and remedying waste waters contaminated with chlorinated compounds ${ }^{[8,9]}$, nitro aromatic compounds $^{[10]}$, nitrates ${ }^{[11]}$ and heavy metals ${ }^{[12]}$, and even for the deoxidization of more complex anthropogenic chemicals including pesticides ${ }^{[13]}$ and dyes ${ }^{[14-19]}$. Among these compounds, azo dyes are easier to be deoxidized than to be oxidized. Thus, deoxidization with ZVI is one of the most important paths of degradation. The degradation mechanism of azo dyes on the surface of ZVI is illustrated in Figure $1^{[17,18]}$.

Though there have been many reports on the treatment and remediation of waste water with ZVI, many pivotal technological problems have to be solved before the actual application. For example, the balance between the ZVI dispersion and treatment capacity, the residual of ZVI and the release of large amounts of $\mathrm{Fe}^{2+}$ in the water remain great challenges, which might be resolved with the promotion of preparation, reclaiming and refreshing technologies of ZVI.

A new method for the transformation of azo dyes into easier bio-decomposition compounds with NZVI has been developed in this study. The NZVI immobilized on the surface of cation exchange resin was prepared and characterized. Then, some factors that influence the degradation efficiency and the reducing ability of the refreshed NZVI were studied. Results show that the refreshed NZVI possess strong activity, and immobilizing NZVI on the surface of cation exchange resin packed in a column successfully solved the problem of reclaiming and refreshing of NZVI.

\section{Experimental section}

\subsection{Chemicals}

All chemicals were used without further purification. Acid Orange 7, 1-amino-2-naphathol hydrochloride, and sulfianilic acid were purchased from Acros (NJ, USA). Acid Orange 8 was purchased from Aldrich (CAS 5850-86-2). Acid Orange 10, Methyl Orange, and Sunset Yellow were all purchased from TCI (Tokyo Kasei Kogeo Co., Ltd.). The structures of the five studied azo dyes are listed below.

Aqueous stock solutions $\left(2.5 \mathrm{~g} \cdot \mathrm{L}^{-1}\right)$ were prepared by dissolving corresponding mass of reagents in $250 \mathrm{~mL}$ water, respectively. The cation exchange resin (exchange capacity $\geqslant 4.2 \mathrm{mmol} \cdot \mathrm{g}^{-1}$, granularity $(0.3-1.2 \mathrm{~mm})$ $\geqslant 95 \%$, water content $46 \%-52 \%$ ) was purchased from Shanghai Curatorial Factory (China). $\mathrm{FeCl}_{2} \cdot 4 \mathrm{H}_{2} \mathrm{O}(99 \%)$ was purchased from Tianjin Reagent Co. Ltd. (China). $\mathrm{KBH}_{4}(99 \%)$ was purchased from Langfang Reagent Co. Ltd. (China).

\subsection{Synthesis of NZVI immobilized on cation ex- change resin}

The cation exchange resin supported NZVI was pre-

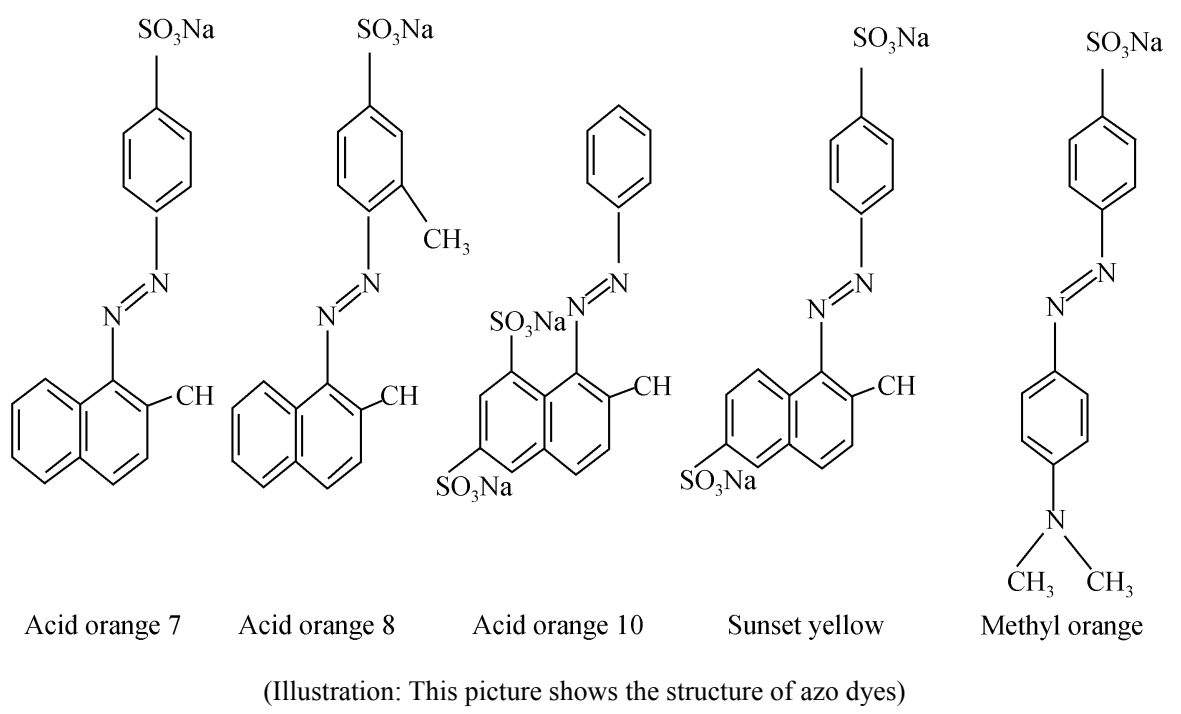



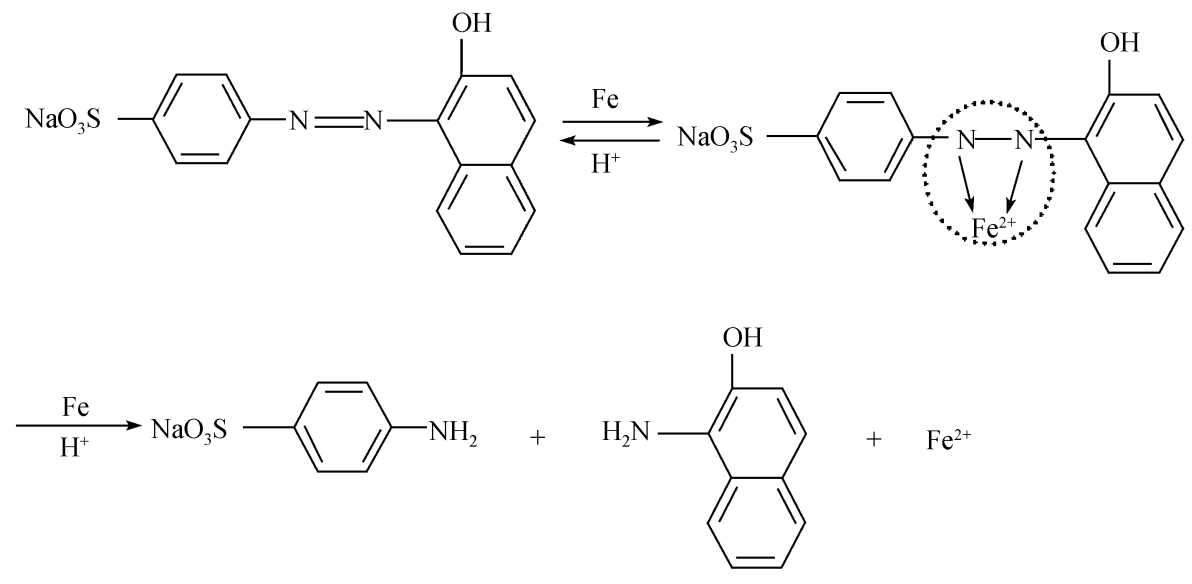

Figure 1 Azo dyes degradation schematic using ZVI.

pared by the method of reducing iron salt in aqueous solutions ${ }^{[20]}$ with some modifications. Briefly, the resin was firstly treated with $4 \mathrm{~mol} \cdot \mathrm{L}^{-1} \mathrm{HCl}$ for $30 \mathrm{~min}$ to replace $\mathrm{Na}^{+}$with $\mathrm{H}^{+}$and washed with plenty of water to remove the residual $\mathrm{HCl} . \mathrm{FeCl}_{2} \cdot 4 \mathrm{H}_{2} \mathrm{O}$ and the treated resin were mixed in aqueous solutions at a 1:5 mass ratio and stirred for $30 \mathrm{~min}$ to ensure that $\mathrm{Fe}^{2+}$ could be exchanged on the surface of the resin, and then the resin was washed again with deionized water to remove the residual $\mathrm{Fe}^{2+}$.

To reduce the $\mathrm{Fe}^{2+}$ to NZVI, $20 \mathrm{~g}$ of the above prepared resin was transferred to a $500 \mathrm{~mL}$ clean beaker containing $100 \mathrm{~mL}$ ice water and the reaction was initiated by slow addition of $100 \mathrm{~mL} \mathrm{KBH} 4$ solution $(0.2$ $\mathrm{mol} \cdot \mathrm{L}^{-1}$, prepared in ice water) under magnetic stirring. The resin quickly transferred to grey from buff, and then to black, coupling with many $\mathrm{H}_{2}$. About 30 min later, the resin was washed again to remove the rest of $\mathrm{KBH}_{4}$ and conserved in weaker basic solutions for use.

\subsection{Surface characterization}

The new NZVI, the used NZVI and the refreshed NZVI were all washed with deionized water to remove the impurities and then dried in $\mathrm{N}_{2}$ at ambient temperature. The characterization was carried out on an S-3000 (Hitachi) scanning electron microscope (SEM) coupled with energy dispersive spectroscope (EDS). The SEM was conducted under the following conditions: Accelerating voltage $15.0 \mathrm{kV}$, working distance $5.3 \mathrm{~mm}$, and high vacuum mode. For EDS, the operating conditions were $15.0 \mathrm{kV}$ accelerating voltage, and $15.0 \mathrm{~mm}$ working distance under high vacuum mode.

\subsection{Degradation in batch system}

$2 \mathrm{~g}$ of the prepared cation exchange resin supported NZVI was added into a group of $50 \mathrm{~mL}$ of $0.05 \mathrm{mg} \cdot \mathrm{mL}^{-1}$ azo dye solutions and the reaction started. After an interval of several minutes, one of them was stopped and the supernatant was collected for analysis by UV-vis spectroscopy.

\subsection{Degradation in packing column system}

The packing column, with inner diameter of $0.6 \mathrm{~cm}$ and length of $15 \mathrm{~cm}$, was packed with the prepared cation exchange resin supported NZVI (about $0.4 \mathrm{~g}$ iron). The initial $200 \mathrm{~mL}$ of azo dye solution $\left(0.05 \mathrm{~g} \cdot \mathrm{L}^{-1}, \mathrm{pH} 5.3\right)$ was pumped with a peristaltic pump through the column, where the degradation reaction happened, at a flow rate of $80 \mathrm{~mL} \cdot \mathrm{min}^{-1}$ and the effluent was directly analyzed for evaluating the degradation efficiency or taken back to the same container for another circle.

\subsection{Activation of NZVI}

The used resin was washed with deionized water to remove the adsorbed impurities and treated with $\mathrm{KBH}_{4}$ solution to reduce $\mathrm{Fe}^{2+}$ to NZVI again. For the column system, refreshing could be realized by pumping $\mathrm{KBH}_{4}$ solution through the packed column under circulatory mode. For evaluating the activity of the refreshed NZVI, the experiments described above (part 1.4 or 1.5) were repeated.

\subsection{Analysis of azo dyes and their degradation products}

All the samples were diluted 10 times before analysis. The concentrations of azo dyes were determined with an 
UV-3000 (Shimazu) spectroscope under the scanning mode. A HPLC-UV (Aglient 1100) equipped with a J'sphere ODS-H80 (S-4 $\mu \mathrm{m}, 8 \mathrm{~nm}, 150 \times 4.6 \mathrm{~mm})$ column was used for the determination of azo dyes and their relevant products. The mobile phase was the mixture of methanol and water $(80+20)$ and the flow rate was $1.0 \mathrm{~mL} \cdot \mathrm{min}^{-1}$.

\subsection{The determination of $\mathrm{Fe}^{2+}$}

$\mathrm{Fe}^{2+}$ concentration, released into the solution from the oxidization of the NZVI in the degradation process of azo dyes, was determined by using the $o$-phenanthroline hydrate- spectrophotometric method.

\section{Results and discussion}

\subsection{Characterization of NZVI immobilized on cation exchange resin}

Figure 2 shows the SEM images and the energy spectrum of NZVI. The left part illustrates that the diameters of the new, used, and refreshed NZVI were about 100$160 \mathrm{~nm}$ and they dispersed evenly on the surface of the resin with many ravines (especially for the new NZVI) which increased the surface area.

The right part is the semi-quantitative information (chosen from the entire eyeshot, not a spot) of the new NZVI, which shows the elemental formation of the material. As the iron layer is so thin that the electron beam can penetrate through it and arrive at the surface of the resin, high contents of $\mathrm{S}, \mathrm{C}$, and $\mathrm{O}$ that were composed of the cation resin was shown in the EDS. $\mathrm{K}$ is ex- changed onto the resin during the NZVI formation process. The energy spectrum indicates that NZVI is the only reducing reagent in the degradation process.

\subsection{Degradation of azo dyes}

Figure 3 illustrates the degradation efficiency of Acid Orange 7 in the batch system at different $\mathrm{pH}$ values. The initial reaction rate is very fast, but it decreases quickly with the increasing of time and shows no obvious change 5 min later. Figure 3 also shows that the decolorization ratio decreases with the increasing of $\mathrm{pH}$. The maximum degradation efficiency is only $70 \%-85 \%$ at $\mathrm{pH} \geqslant 6.2$, but it could increase and even reach up to $100 \%$ with the addition of acids into the system. Therefore, it can be concluded that $\mathrm{H}^{+}$is one of the reactants. This result agreed with the reaction mechanism shown in Figure 1, i.e., the azo dyes adsorbed onto the surface of NZVI interact with $\mathrm{H}^{+}$to form a transitional product, which is then transformed into the terminal product (amine compounds) under the interaction of another $\mathrm{H}^{+[17,18]}$.

Figure 4(a) shows the decolorization ratio of azo dyes with the increase of reaction time: Acid Orange 8 had the highest rate, followed by Sunset Yellow and Acid Orange 7, while Acid Orange 10 and Methyl Orange had the lowest rates. Among the five azo dyes, Acid Orange 10 and Methyl Orange are the most hydrophobic (see molecular structure in Figure 1) and their products have lower aqueous solubility. This may result in the relatively low diffusing away rate and thus higher content of terminal products on the NZVI surface, which reduced the reaction rates from transitional products to terminate
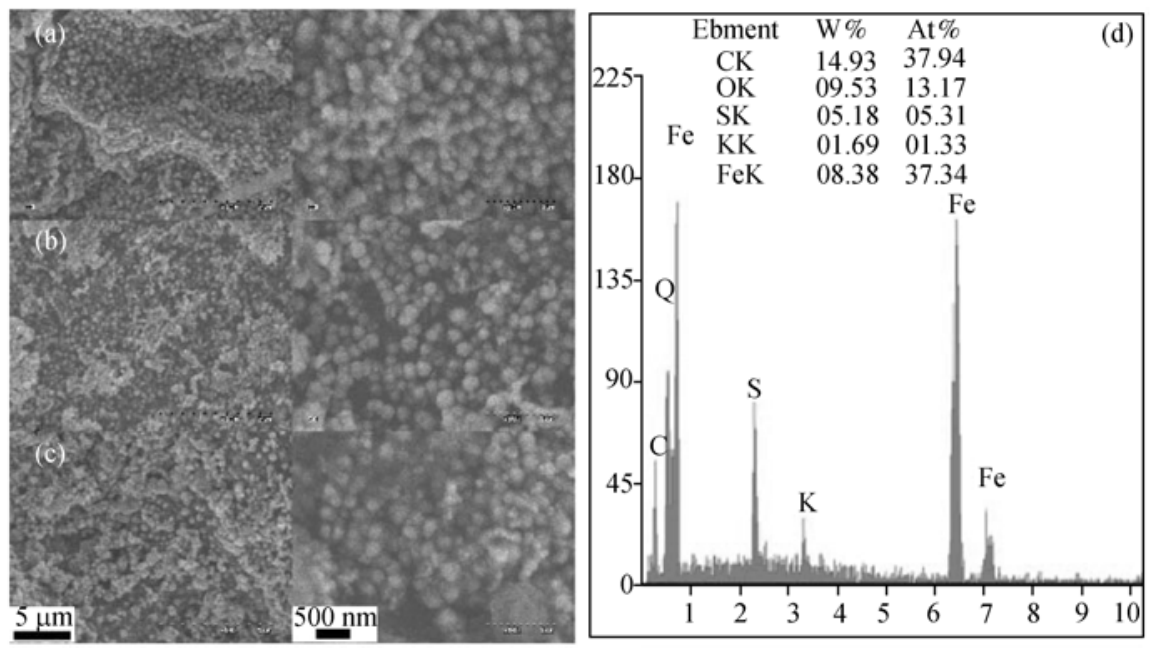

Figure 2 Scanning electron microscopy (SEM) image and EDS of NZVI. (a) New NZVI; (b) used NZVI; (c) refreshed NZVI; (d) energy dispersive spectroscopy (EDS) spectrum of new NZVI. 


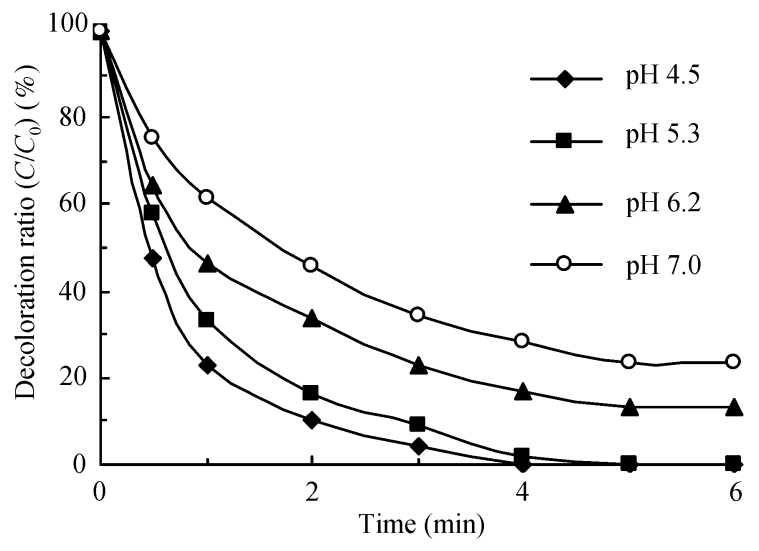

Figure 3 Effect of $\mathrm{pH}$ on the decolorization of Acid Orange 7. Concentration: $0.05 \mathrm{~g} \cdot \mathrm{L}^{-1}$; Mass of as-prepared material: $2 \mathrm{~g}$ (wet weight).

products. Although Acid Orange 8 has lower hydrophilicity than Acid Orange 10 and Methyl Orange, it still had the highest decolorization rate, and up to $97 \%$ of Acid Orange 8 was decolorized in $4 \mathrm{~min}$. This is because the methyl radical of Acid Orange 8 has stronger ability of electron offering that accelerates the formation of the transitional product of Acid Orange 8 with iron.

In the packing column system, the degradation rate was influenced not only by the solution acidity, but also by the flow rate and the design of the column. Figure 4(b) shows the increase of decolorization ratio with increasing circular times of the azo dye solutions. As can be seen, Acid Orange 10 and Methyl Orange still had the lowest decolorization ratio, but Acid Orange 8 had lower decolorization ratio than that of Acid Orange 7 and Sunset Yellow. After the 1st circle, the decolorization ratio was $79 \%$ for Acid Orange 8, 89\% for Acid Orange 7 , and $88 \%$ Sunset Yellow, which were lower than that in the batch system. Probably in the column system, the reactants and products were taken away quickly which decreased the reaction time and thus the decolorization ratio. At a fixed flow rate, the compounds with higher adsorption capacity can stay on the NZVI in the packing column for a longer time that promoted the formation of the transitional and terminal products. Therefore, the adsorption of azo dyes on the surface of ZVI is also an important factor that affects the degradation rate in the packing column. Figure 4(b) also indicates that the decolorization had no significant change after 2 circles. This is because the consumption of $\mathrm{H}^{+}$in the solution, and the degradation ratio decreased very quickly with the acidification of the solution. Obviously, this result accorded with the mechanism shown in Figure 3 and further demonstrated that $\mathrm{H}^{+}$is one of the reactants.

\subsection{Mass balance in the decolorization process}

It is well known that nano-materials have a large ratio of surface area to volume and strong adsorption capacity for reactants and products. If the degradation ratio of a compound is calculated based on the mass difference of the reactant mass before and after the reaction, the adsorption mass will be regarded as the degradation mass and results in positive error. In order to investigate influence of the adsorption on the calculation of degradation ratio, the mass of reactants and products are determined and calculated according to their molar ratio. Figure 5(a) shows the mass balance of Acid Orange 10 in the batch system. From the total mass curve it is clear that the adsorption did exist at the beginning of reaction and reached up to $18 \%$ in $1 \mathrm{~min}$, then it decreased gradually and disappeared finally. Figure 5(b) and 5(c) shows mass balance of Acid Orange 10 and Acid Orange 7 in the packing column system. After the 1st circle, the adsorption mass of Acid Orange 10 and Acid Orange 7 was $12 \%$ and $17 \%$, respectively. The adsorption almost
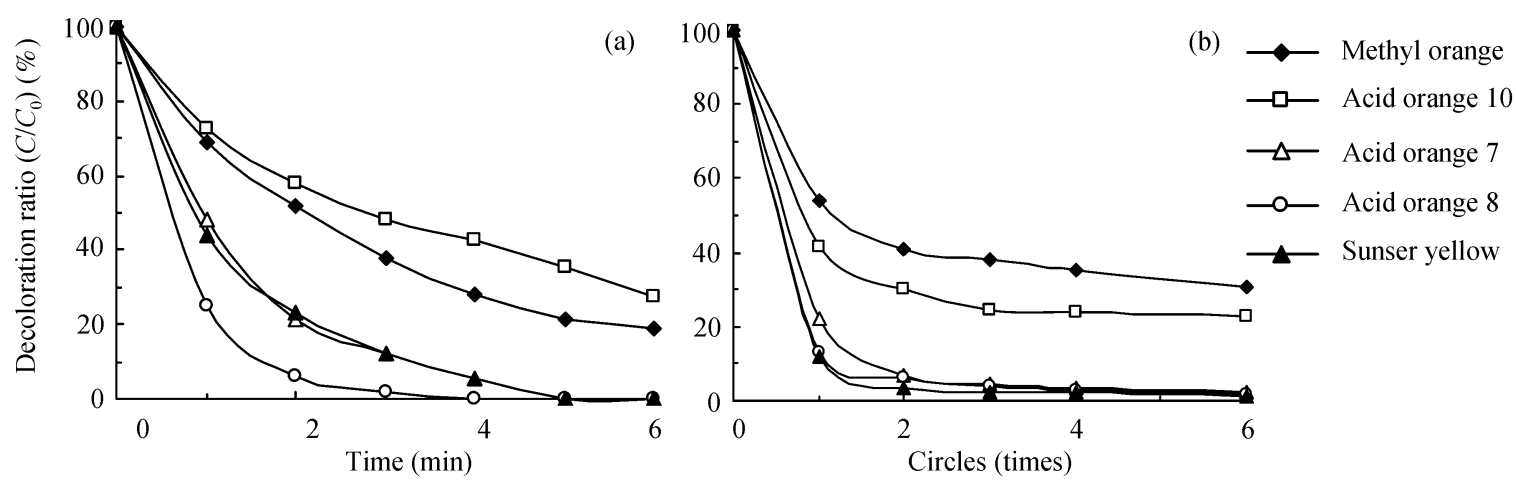

Figure 4 Decolorization ratio of the five azo dyes. (a) Batch system; (b) packing column system. 

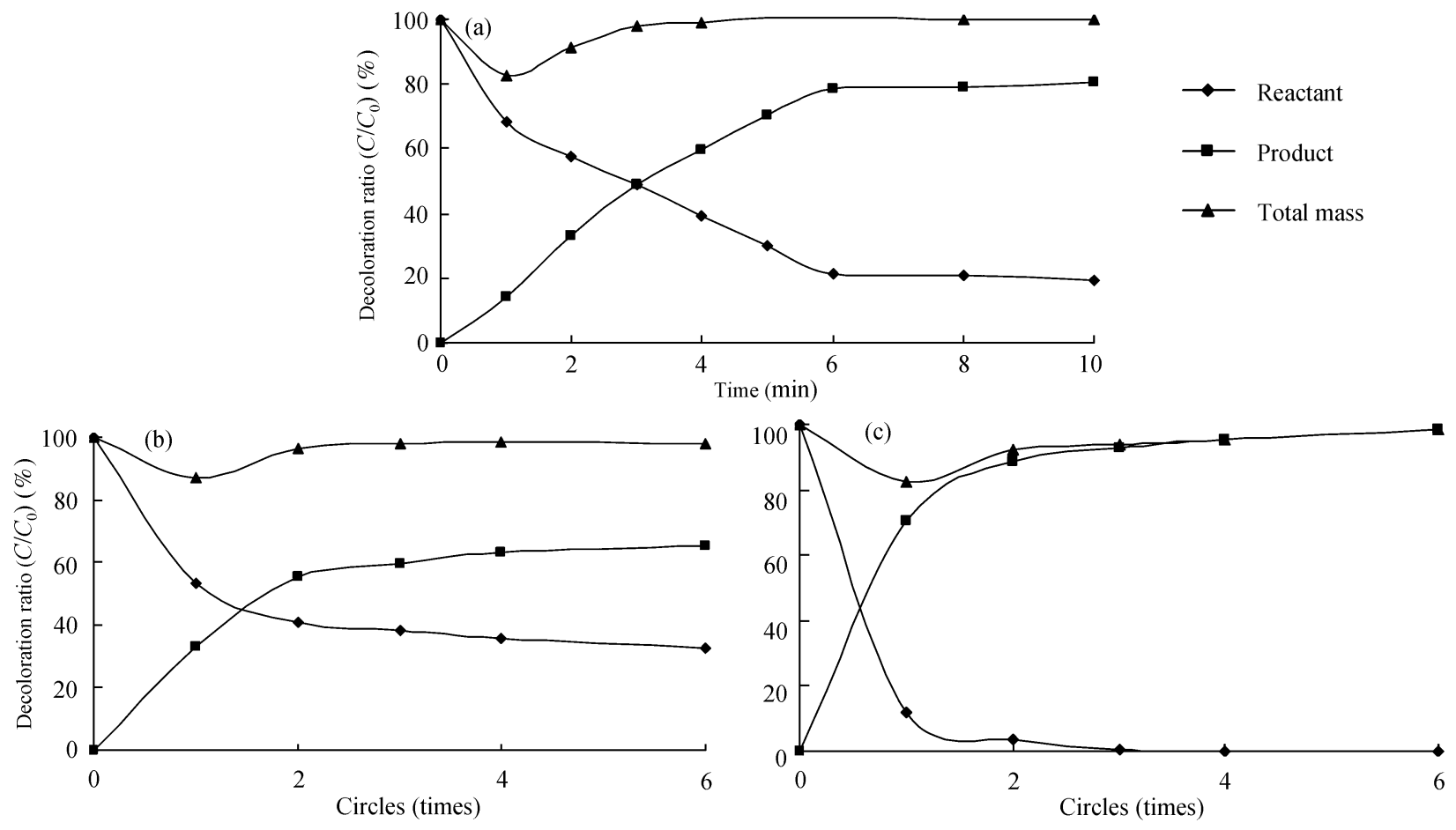

Figure 5 Mass balance curve. (a) Acid Orange 10(Batch system); (b) Acid Orange 10(Column System); (c) Acid Orange 7(Column system).

disappeared from the 2nd circle for Acid Orange 10, whereas it existed all the while and decreased slowly in the following circles for Acid Orange 7. The results of these two systems indicate that the decolorization mainly resulted from the deoxidization of ZVI instead of adsorption.

Figure 5(b) and Figure 5(c) also show the different adsorption ability of NZVI for the two azo dyes. This adsorption ability depends not only on the hydrophilicity and the spacial structure of azo dyes, but also on the positive charge on the surface of NZVI resulting from the hydrogen bond interaction between the metal hydroxy groups of NZVI and $\mathrm{H}^{+}$. For a packed column system with fixed acidity and flow rate, the larger the mass of the adsorbed azo dyes on the surface of NZVI, the higher the odds of formation of transitional and terminal products, which results in higher decolorization ratio. Thus, adsorption can not only accelerate decolorization, but also promote decomposition.

\subsection{Refreshing of NZVI}

All the refreshed NZVI can quickly decompose the azo dyes (see Figure 6), though the decolorization efficiency decreased slightly with the increasing of refreshing times. Even after the 10th refreshing, the decomposition efficiency of the refreshed NZVI was $90 \%$ of that of the

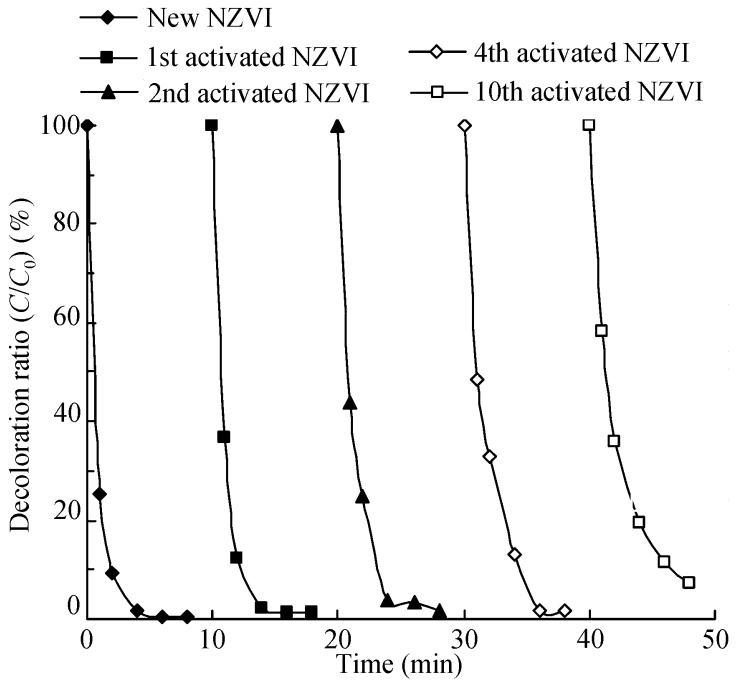

Figure 6 Activity of the refreshed NZVI.

new NZVI, and satisfactory decomposition ratio was obtained in $8 \mathrm{~min}$.

With the mixing of NZVI and excess amount of dyes, the $\mathrm{Fe}^{2+}$ concentration increased with the time increasing and then kept constant until all the NZVI were consumed, as shown in Figure 7. This is because most of the $\mathrm{Fe}^{2+}$, released from the oxidization of NZVI, are exchanged onto the surface of the resin and only small amounts stay in the aqueous solution. Thus, when the 
resin with $\mathrm{Fe}^{2+}$ is reduced with $\mathrm{KBH}_{4}$ after the decomposition reaction, NZVI is formed again and it is ready for decomposing azo dyes. There is no obvious difference between the new and the refreshed NZVI in the micro-morphology, i.e., the refreshed NZVI is still spheres with ravines (See Figure 2(c)). The resin acts as both the supporter of NZVI and the collector of $\mathrm{Fe}^{2+}$, which obviously reduces $\mathrm{Fe}^{2+}$ release and its potential negative effect on the environment.

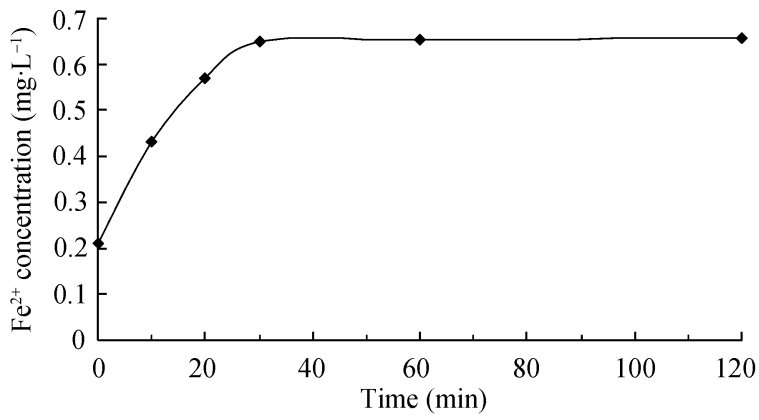

Figure $7 \quad \mathrm{Fe}^{2+}$ released into the aqueous solution. NZVI immobilized on resin ( $2 \mathrm{~g}$ of wet weight, with about $\left.0.1 \mathrm{~g} \mathrm{Fe}^{0}\right)$ and excess azo dyes were mixed in $50 \mathrm{~mL}$ solution volume.

1 Tan B H, Teng T T, Omar A K M. Removal of dyes and industry dye wastes by magnesium chloride. Water Res, 2000, 34(2): 597-601

2 Van der Bruggen B, De Vreese I, Vandecasteele C. Water reclamation in the textile industry: Nanofilitration of dye baths for wool dyeing. Ind Eng Chem Res, 2001, 40(18): 3973-3978

3 Feng J Y, Hu X J, Yue P L, Zhu H Y, Lu G Q. Degradation of Azo-dye Orange II by a photoassited fenton reaction using a novel composite of iron oxide and silicate nanoparticles as a catalyst. Ind Eng Chem Res, 2003, 42(10): 2058-2066

4 Li M Y, Xiong L, Chen Y Y, Zhang N, Zhang Y M, Yin H. Studies on photo-electro-chemical catalytic degradation of acid scarlet 3R dye. Sci China Ser B-Chem (in Chinese), 2005，35(2): 144-150

5 Li J Y, Chen C C, Zhao J C, Zhu H Y, Ding Z. Photo degradation of dye on the bentonite impregnated $\mathrm{TiO}_{2}$ under $\mathrm{UV}$ irradation. Sci China Ser B-Chem (in Chinese), 2002, 32(3): 268-270

6 Khan A A, Husain Q. Decolorization and removal of textile and non-textile dyes from polluted wastewater and dyeing effluent by using potato (Solanum tuberosum) soluble and immobilized polyphenol oxidase. Bioresource Technol, 2007, 98 (5): 1012-1019

7 Scott J P, Ollis D F. Integration of chemical and biological oxidation process for water treatment: Review and recommendations. Environ Prog, 1995, 14(2): 88-103

8 Liu Y, Majetich S A, Tilton R D, Sholl D S, Lowry G V. TCE dechlorination rates, pathways, and efficiency of nanoscale iron particles with different properties. Environ Sci Technol, 2005, 39(5): $1338-1345$

9 Cheng R, Wang J L, Zhang W X. The research process on degradation of halogenated organic compounds by nano iron. Process Chem, 2006, 18(1): 93-99

\section{Conclusion}

NZVI immobilized on the surface of cation exchange resin was synthesized, and their applications in the degradation of azo dyes were studied in both the batch system and the packing column system. It was found that $0.05 \mathrm{~g} \cdot \mathrm{L}^{-1}$ of azo dyes can be reduced to a very low level in several minutes or circles. The decomposition efficiency of azo dyes depends strongly on structure of dyes, $\mathrm{pH}$ reaction solution, and adsorption of reactants. It was demonstrated that the decomposing efficiency increases with hydrophilicity, $\mathrm{H}^{+}$is one of the reactants, and adsorption promotes decomposition. Furthermore, $\mathrm{Fe}^{2+}$ can be exchanged onto the surface of the cation exchange resin, the supporter of NZVI, during the decomposition process, and the $\mathrm{Fe}^{2+}$ on the resin can be reduced to form refreshed NZVI for reusing. Thus, decomposing pollutants in aqueous solution with columns, packed with NZVI immobilized on the cation exchange resin, is a promising technology that can solve the reclaiming and refreshing problem of NZVI.

We appreciate the constructive comments from an anonymous reviewer

10 Agrawal A, Tratnyek P G. Reduction of nitro aromatic compounds by zero-valent iron mental. Environ Sci Technol, 1996, 30(1): 153-160

11 Huang C P, Wang H W, Chiu P C. Nitrate reduction by metallic iron. Water Res, 1998, 32(8): 2257-2264

12 Fiedor J N, Bostick W D, Jarabek R, Farrell J. Understanding the mechanism of uranium removal from groundwater by zero-valent iron using X-ray photoelectron spectroscopy. Environ Sci Technol, 1998, 32(10): $1466-1473$

13 Eykholt G R, Davenport D T. Dechlorination of the chloroacetanilide herbicides alachlor and metolachlor by iron metal. Environ Sci Technol, 1998, 32(10): 1482-1487

14 Nam S, Tratnyek P G. Reduction of azo dye with zero-valent iron Water Res, 2000, 34(6): 1837-1845

15 Bigg T, Judd S J. Kinetics of reductive degradation of azo dye by zero-valent iron. Process Saf Environ, 2001, 79(B5): 297-303

16 Perey J R, Chiu P C, Huang C P, Cha D K. Zero-valent iron pretreatment for enhancing the biodegradability of azo dyes. Water Environ Res, 2002, 74(3): 221-225

17 Cao J, Wei L, Huang Q, Han S. Reducing degradation of azo dyes by zero-valent iron in aqueous solution. Chemosphere, 1999, 36(3) $565-571$

18 Weber E J. Iron-mediated reductive transformation: Investigation of reaction mechanism. Environ Sci Technol, 1996, 30(2): 716-719

19 Pereira W S, Freire R S. Azo dye degradation by recycled waste zero-valent iron powder. J Brazil Chem Sci, 2006, 17(5): 832-838

20 Li F, Vipulanandan C, Mohanty K K. Microemulsion and solution approaches to nanoparticle iron production for degradation of trichloroethylene. Colloid Surface A, 2003, 223: 103-112 